\title{
Mensch und Maschine Hand in Hand
}

\begin{abstract}
„Die Fabrik der Zukunft wird zwei Angestellte haben: einen Menschen und einen Hund. Der Mensch ist dazu da, den Hund zu füttern. Der Hund wird die Aufgabe haben, den Menschen davon abzuhalten, die voll automatisierten Geräte anzufassen“, so Warren G. Bennis (1925-2014). Professor Bennis war ein herausragender US-amerikanischer Wirtschaftswissenschaftler und ein führender Autorität im Bereich Organisationsentwicklung, Führungstheorie und Änderungsmanagement. Dieses humorvolle Zitat konnte vor ca. 30 Jahren durchaus als düstere Prophezeiung für die Fabrik der Zukunft angesehen werden. Heute sind die Erwartungen, die Wahrnehmung und der Glaube an die Umsetzung von Automatisierungslösungen - geprägt durch Industrie 4.0 - deutlich positiver. Es wurden erfolgreich enorme Anstrengungen unternommen, um Konzepte für eine vernetzte und global agierende Produktion zu realisieren (Europa, Industrie 4.0; China, Made in China 2025; USA, Advanced Manufacturing; Japan, Super Smart Society).

Eine sehr große Herausforderung und Einschränkung ist in allen Fällen jedoch die Balance zwischen Mensch und Maschine. Durch Automatisierung, Künstliche Intelligenz und neuartige Sensoren wurden aufgrund der genannten Aktivitäten ein innovativer Produktions- und Distributionsprozess geschaffen. Dabei werden Prozesse durchgehend digitalisiert und Roboter zur eigenständigen oder kollaborierenden Automatisierung ertüchtigt, die schließlich durch den Menschen an Leitständen überwacht werden. Die zugehörigen Fertigungsanlagen sind aufeinander abgestimmt sowie synchronisiert und gewährleisten dabei höchste Effizienz und Effektivität.

In Zukunft wird die Synergie der Fähigkeiten von intelligenten Maschinen und Menschen noch wichtiger sein, um Produktivität und Qualität weiter zu steigern und gleichzeitig sehr gute Arbeitsbedingungen für den Menschen zu sichern. Dies wird zu Fertigungsszenarien motivieren, in denen Menschen und industrielle Geräte im selben Bereich arbeiten und gegenseitig von ihren Fähigkeiten profitieren.
\end{abstract}

Ihre ZWF-Herausgeber und-Redaktion

Über MPDV

MPDV ist der Marktführer für IT-Lösungen in der Fertigung. Mit mehr als 40 Jahren Projekterfahrung im Produktionsumfeld verfügt MPDV über umfangreiches Fachwissen und unterstützt Unternehmen jeder Größe auf ihrem Weg zur Smart Factory. Produkte von MPDV wie das Manufacturing Execution System (MES) HYDRA, das Advanced Planning and Scheduling System (APS) FEDRA oder die Manufacturing Integration Platform (MIP) ermöglichen es Fertigungsunternehmen, ihre Produktionsprozesse effizienter zu gestalten und dem Wettbewerb so einen Schritt voraus zu sein.
In Echtzeit lassen sich mit den Systemen fertigungsnahe Daten entlang der gesamten Wertschöpfungskette erfassen und auswerten.

Kontakt:

MPDV Mikrolab GmbH

Jürgen Petzel

Römerring 1

74821 Mosbach

Tel.: +49 $62619209-101$

Fax: +49 626118139

info@mpdv.com

www.mpdv.com

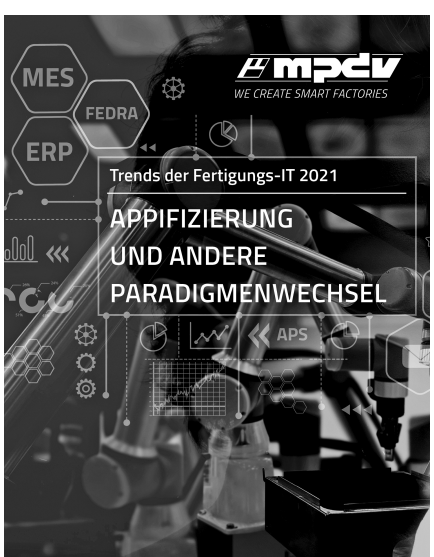

